\title{
Shedding Light on the Role of Ventral Tegmental Area Dopamine in Reward
}

\author{
Benjamin T. Saunders and Jocelyn M. Richard \\ Department of Psychology (Biopsychology Program), University of Michigan, Ann Arbor, Michigan 48109 \\ Review of Adamantidis et al.
}

Successful reward seeking requires the interaction of multiple psychological processes, including learning (i.e., creating connections between actions/stimuli and rewarding outcomes), motivation (i.e., wanting to obtain rewarding outcomes), and affect (i.e., liking rewarding outcomes). There are two primary forms of reward learning, instrumental and Pavlovian (Cardinal et al., 2002; Berridge and Robinson, 2003). Instrumental learning involves the establishment of associations between actions and the outcomes they produce: actions are reinforced when they produce rewarding outcomes. In contrast, Pavlovian learning involves the formation of associations between stimuli/cues and the outcomes they predict. These learning processes are dissociable but, critically, Pavlovian learning affects actions as well: environmental stimuli associated with reward acquire motivational value of their own, meaning they can instigate approach behavior, elicit consumption of rewards, reinforce actions, and/or invigorate ongoing actions. Finally, the affective components of rewards shape learning by reinforcing actions and/or by increasing the

Received Sept. 27, 2011; revised 0ct. 23, 2011; accepted 0ct. 25, 2011. This work was supported by National Research Service Award Fellowships DA030801 (B.T.S.) and MH090602 (J.M.R.). We thank Terry Robinson and Kent Berridge for helpful comments.

Correspondence should be addressed to Benjamin T. Saunders, Department of Psychology (Biopsychology Program), University of Michigan, East Hall, 530 Church Street, Ann Arbor, MI 48109. E-mail: btsaunde@umich.edu.

DOI:10.1523/JNEUROSCI.4924-11.2011

Copyright $\odot 2011$ the authors $\quad 0270-6474 / 11 / 3118195-03 \$ 15.00 / 0$ motivational value of reward-associated stimuli.

Different types of learning and motivational processes that underlie reward seeking are thought to be neurobiologically dissociable (Cardinal et al., 2002; Berridge and Robinson, 2003; Fields et al., 2007). Converging lines of research suggest that dopaminergic neurons within the ventral tegmental area (VTA), via projections onto forebrain structures such as the nucleus accumbens, prefrontal cortex, and amygdala, play a key part in Pavlovian learning and motivation, and in the expression of learned appetitive behaviors in general. Dopamine (DA) is thought to be less important for instrumental learning, however (Fields et al., 2007; Tsai et al., 2009; Flagel et al., 2011; Wassum et al., 2011). Activation of DA neurons has been suggested to have positive reinforcing properties, because pharmacological or electrical stimulation tends to facilitate reward seeking, whereas inhibition or lesion tends to reduce reward seeking (Cheer et al., 2007; Fields et al., 2007). Many studies that address the role of dopamine in instrumental learning fail, however, to adequately dissociate learning from motivation and/or fail to remove Pavlovian cue influences from instrumental reward-seeking paradigms. Collectively, the psychological processes underlying reward seeking are complex, and as such, studies must carefully isolate these processes to determine DA's specific role in reward.

Until recently, technological barriers made it impossible to specifically manipu- late DA neurons to test their causal contribution to reward. In a recent issue of The Journal of Neuroscience, Adamantidis et al. (2011) overcame this barrier by using in vivo optogenetic techniques to selectively stimulate DA neurons within the VTA during different phases of a food-seeking task and in a self-stimulation paradigm. To target DA neurons, they infused a virus for Credependent expression of the light-activated channelrhodopsin-2 (ChR2) into the VTA of mice expressing Cre specifically in tyrosine hydroxylase-positive (i.e., DAproducing) neurons. This resulted in the targeted expression of ChR2 in only those cells. A similar procedure was previously shown to produce ChR2 expression in 90\% of DA neurons near the injection site, with high specificity (Tsai et al., 2009).

In their first experiment, Adamantidis et al. (2011) tested whether optogenetic activation of VTA DA neurons would bias responding during the acquisition phase of a food-seeking task. Pressing an "active" lever resulted in the simultaneous delivery of a food pellet, de-illumination of a cue light above the lever, and stimulation of DA neurons. Inactive-lever presses were followed by food delivery and de-illumination of a different cue light. Over several training sessions, ChR2 mice developed a preference for pressing the active lever, eventually showing a clear discrimination compared with control mice (Adamantidis et al., 2011, their Fig. 3 ). The authors concluded that optical activation of VTA DA neurons has positive reinforcing properties. 
We suggest that reinforcement is an inappropriate description of the effect, however, as there is no evidence for a direct DA contribution to reinforcement. Critically, results from this experiment are difficult to interpret because the design does not allow a clear judgment of what role DA plays in the establishment of active lever preference. Given that reward delivery was paired with an action (lever press) and cue (light de-illumination/lever), instrumental and Pavlovian learning are confounded in the task. As we discuss above, Pavlovian learning could be expected to increase the mice's motivation to press the paired lever. Therefore, it is possible that optical DA stimulation may have directly reinforced pressing of the active lever, or enhanced the motivational value of the light/lever cues. Additionally, DA stimulation might have affected the mice's desire to eat, thus changing the food's value. Interestingly, as a consequence of making more active-lever presses, ChR2 mice earned more food pellets than controls, but did not consume significantly more pellets. This indicates that $\mathrm{ChR} 2$ mice left some pellets unconsumed, suggesting the facilitation of active-lever pressing by optical stimulation of DA neurons was not driven by an enhancement of the affective value or palatability of the reward itself. Nonetheless, the dopamine effect may have been driven at least in part by enhancement of the motivational value of the cues associated with the reward. This is consistent with a recent report showing that systemic dopamine antagonism does not impair instrumental incentive learning, where the value of a reward is changed after a motivational shift induced by food deprivation (Wassum et al., 2011).

Adamantidis et al. (2011) next examined the ability of optical stimulation of VTA DA neurons to reinstate extinguished food-seeking behavior. In this phase, active-lever presses resulted in optical stimulation, but no food or cue light de-illumination. Interestingly, ChR2 mice significantly renewed pressing the active lever (Adamantidis et al., 2011, their Fig. 4). An intriguing possibility suggested by this result is that through its association with active-lever presses, cue-light deillumination, or food during acquisition training, the optically evoked DA signal acquired the ability to motivate behavior, effectively becoming a conditioned reinforcer, i.e., something for which ChR2 mice were willing to work. Given that Adamantidis et al. (2011) found that optical stimulation of DA neurons in naive mice was insufficient to reinforce lever presses (see below), we might expect the conditioned reinforcing properties of DA stimulation to eventually extinguish in the absence of primary reward, a possibility that could be tested using a longer reinstatement phase.

In a separate set of experiments, Adamantidis et al. (2011) investigated the ability of optical stimulation of VTA DA neurons to support instrumental responding in the absence of food reward using a procedure similar to electrical intracranial self-stimulation. In this paradigm, active-lever presses were followed only by optical stimulation of the VTA. ChR2 mice did not develop a preference for the active lever (Adamantidis et al., 2011, their Fig. 3). Importantly, this suggests that optical activation of VTA DA neurons in the absence of reward or reward-paired cues does not reinforce behavior. This possibility is especially interesting given that electrical stimulation of the VTA supports robust instrumental responding (Cheer et al., 2007). The authors previously demonstrated that optical activation of VTA DA neurons is sufficient to produce a conditioned place preference (Tsai et al., 2009). However, the place preference paradigm involves Pavlovian learning, rather than instrumental learning. Thus, the results of Tsai et al. (2009) demonstrate that a phasic increase in VTA DA neuron activity is sufficient to form a Pavlovian association and assign motivational value to cues.

Although the data in Adamantidis et al. (2011) suggest that VTA DA activation does not directly reinforce behavior, it may do so under different experimental conditions. Dopamine neurons are frequently treated as a homogeneous population in the context of explaining behavioral effects. Yet increasing evidence suggests that VTA DA neurons are heterogeneous with regard to their neuroanatomical targets, physiological properties, and responses to salient appetitive versus aversive stimuli (Fields et al., 2007; Lammel et al., 2011). This should be considered when interpreting the negative self-stimulation effect reported by Adamantidis et al. (2011). No data are given on the pattern and extent of ChR2 expression in the VTA; thus, it is possible that an insufficient number of DA cells were recruited to produce a signal strong enough to support self-stimulation behavior. Additionally, while different DA projection cells are dispersed throughout the VTA, there is some segregation (Lammel et al., 2011), making the characteristics of virus expression important. It is also notable that the optical stimulation parameters used by Tsai et al.
(2009) (50 Hz; 25 pulses; $15 \mathrm{~ms}$ width) to produce a conditioned place preference are considerably different from those used by Adamantidis et al. (2011) (25 Hz; 20 pulses; $5 \mathrm{~ms}$ width). One possibility, therefore, is that the stimulation parameters used by Adamantidis et al. (2011) are nonoptimal or inappropriate for robust self-stimulation.

In conclusion, the results of Adamantidis et al. (2011) suggest that optical stimulation of VTA DA neurons is sufficient to increase motivation to seek out food reward, and it appears to do so by amplifying the motivational value of cues associated with the reward experience, rather than by directly reinforcing actions. Furthermore, through conditioning, optical VTA DA stimulation becomes a signal animals are later willing to work for. While these results provide evidence supportive of recent studies suggesting that DA does not play a major role in instrumental incentive learning (Wassum et al., 2011), they do not clarify potential causal roles DA may play specifically in Pavlovian learning mechanisms. Going forward from this research, it will be important to develop experiments that (1) carefully examine DA's role in instrumental versus Pavlovian learning while isolating learning mechanisms from motivational ones and (2) investigate the contribution of specific DA neuron subpopulations to different reward processes. Optogenetics provides a powerful tool to interrogate neural systems with temporal precision and cell-type specificity to investigate the function of neurons defined by their wiring within a circuit (Yizhar et al., 2011). As such, we hope future studies incorporate sophisticated psychological approaches with these cutting-edge techniques to investigate reward.

\section{References}

Adamantidis AR, Tsai HC, Boutrel B, Zhang F, Stuber GD, Budygin EA, Touriño C, Bonci A, Deisseroth K, de Lecea L (2011) Optogenetic interrogation of dopaminergic modulation of the multiple phases of reward-seeking behavior. J Neurosci 31:10829-10835.

Berridge KC, Robinson TE (2003) Parsing reward. Trends Neurosci 26:507-513.

Cardinal RN, Parkinson JA, Hall J, Everitt BJ (2002) Emotion and motivation: the role of the amygdala, ventral striatum, and prefrontal cortex. Neurosci Biobehav Rev 26:321-352.

Cheer JF, Aragona BJ, Heien ML, Seipel AT, Carelli RM, Wightman RM (2007) Coordinated accumbal dopamine release and neural activity drive goal-directed behavior. Neuron 54:237-244.

Fields HL, Hjelmstad GO, Margolis EB, Nicola SM (2007) Ventral tegmental area neurons in learned appetitive behavior and positive reinforcement. Annu Rev Neurosci 30:289-316. 
Flagel SB, Clark JJ, Robinson TE, Mayo L, Czuj A, Willuhn I, Akers CA, Clinton SM, Phillips PE, Akil H (2011) A selective role for dopamine in stimulus-reward learning. $\mathrm{Na}-$ ture 469:53-57.

Lammel S, Ion DI, Roeper J, Malenka RC (2011) Projection-specific modulation of dopamine neuron synapses by aversive and rewarding stimuli. Neuron 70:855-862.

Tsai HC, Zhang F, Adamantidis A, Stuber GD, Bonci A, de Lecea L, Deisseroth K (2009) Phasic firing in dopaminergic neurons is sufficient for behavioral conditioning. Science 324:1080-1084.

Wassum KM, Ostlund SB, Balleine BW, Maid- ment NT (2011) Differential dependence of Pavlovian incentive motivation and instrumental incentive learning processes on dopamine signaling. Learn Mem 18:475-483.

Yizhar O, Fenno LE, Davidson TJ, Mogri M, Deisseroth K (2011) Optogenetics in neural systems. Neuron 71:9-34. 